\title{
IMPACTOS PSICOSSOCIAIS DO CONTEXTO DE CONSTRUÇÃO DO COMPLEXO PETROQUÍMICO DO RIO DE JANEIRO
}

\author{
PSYCHOSOCIAL IMPACTS IN THE CONTEXT OF THE CONSTRUCTION OF THE RIO DE JANEIRO \\ PETROCHEMICAL COMPLEX
}

\author{
IMPACTOS PSICOSOCIALES DEL CONTEXTO DE CONSTRUCCIÓN DEL COMPLEJO PETROQUÍMICO \\ DE RÍO DE JANEIRO
}

\author{
Marcela de Abreu Moniz ${ }^{1}$ \\ Jaqueline Manhães Pereira ${ }^{2}$ \\ Rayara Mozer Dias ${ }^{3}$
}

Resumo O estudo objetivou descrever a percepção de graduandos de enfermagem sobre impactos psicossociais e estratégias de coping de uma população vulnerabilizada pelo contexto de construção do Complexo Petroquímico do Rio de Janeiro. Tratou-se de pesquisa-ação, descritiva, de abordagem qualitativa, realizada com 18 graduandos de enfermagem por meio da aplicação das técnicas fotovoz e grupo focal durante o mês de maio de 2015. Os dados receberam tratamento analítico de conteúdo. Os participantes destacaram apenas o consumo e tráfico de drogas, a violência sexual, a disponibilidade dos serviços de saúde como impactos sociais com grande potencial de produzir problemas psicológicos, tais como ansiedade e depressão na população, como consequências do processo de construção da indústria. Estratégias de coping aos estressores ambientais focadas na ação coletiva foram problematizadas por apenas dois participantes. Concluiu-se que a inclusão de modo transversal da temática socioambiental - e sua relação com a saúde mental de populações vulnerabilizadas pelos contextos de construção de empreendimentos industriais no país, em disciplinas da graduação e outros espaços acadêmicos - é fundamental, a fim de estimular a capacidade crítica, reflexiva e política de futuros enfermeiros sobre a relação entre projetos de desenvolvimento, degradação ambiental, equidade e impactos psicológicos na população.

Palavras-chave saúde mental; saúde ambiental; percepção; estudantes de enfermagem.
Abstract The study aimed to describe the views nursing students had on the psychosocial impacts and on the coping strategies of a population made vulnerable in the context of the construction of the Petrochemical Complex in Rio de Janeiro, Brazil. It was a descriptive research action using a qualitative approach and including 18 nursing students by means of the application of photovoice techniques and focus groups during the month of May 2015. The data were subjected to analytical content treatment. As consequences of the plant's construction process, participants only highlighted drug consumption and trafficking, sexual violence, and the availability of health services as social impacts with great potential to lead to psychological issues in the population, such as anxiety and depression. Only two participants problematized strategies to cope with environmental stressors focused on collective action. It was concluded that including a cross-section mode of the socio-environmental theme - and its relation to the mental health of populations made vulnerable by the industrial project construction contexts in the country, in undergraduate courses and other academic spaces - is essential to encourage the critical, reflective, and political skills of future nurses on the relationship between development projects, environmental degradation, equity and psychological impact on the population.

Keywords mental health; environmental health; perception; nursing students. 


\section{Introdução}

As dimensões físicas, biológicas e sociais do ambiente medeiam a percepção, as sensações e as atitudes dos indivíduos e grupos diante desse mesmo ambiente. Cada pessoa percebe, avalia e interage em seu ambiente, assim como cada ambiente particular exerce efeitos sobre as condutas humanas. Essa inter-relação humana com o ambiente tem sido profundamente abalada nos últimos três séculos, a partir dos impactos de deterioração e desequilíbrio vital que têm sido gerados em ambos em decorrência dos processos de industrialização e modernização tecnológica (Loureiro, 2012; Hassan et al., 2005).

A grande velocidade de trocas de fluxos de matérias, energias e informações produzidas pelas tecnologias tem demandado adaptação e acompanhamento pelo cérebro humano. As relações inter-humanas do homem com o ambiente têm impactado intensamente a saúde mental de indivíduos e comunidades. A acentuação das desigualdades socioambientais provocadas pelo modelo de desenvolvimento capitalista e sua vasta produção tecnológica têm produzido sofrimento humano, medo e estresse coletivo (Seixas e Renk, 2011).

Desse modo, é preciso compreender como indivíduos e coletividades enfrentam problemas socioambientais antigos e respondem a eles - como pequena oferta de serviços de saneamento ambiental, de educação e saúde e também novos - por exemplo, mesma disponibilidade e capacidade desses equipamentos urbanos para atender a um aumento da demanda populacional resultante de processos de construção de grandes empreendimentos.

Os enfrentamentos individuais e coletivos ante os estressores ambientais são traduzidos conforme a formulação de mecanismos psicológicos adaptativos. Enfrentamento ou coping é um interativo processo caracterizado por percepção ou atitude envolvida no sentido de reduzir o fator estressor, se resignar diante desse fator ou negá-lo, dependendo das características da exposição, do indivíduo, da capacidade comunitária e da rede social (Luginaah et al., 2002; Dejours, 2015). Fatores ou condições físicas ou ambientais operam como estressores na medida em que eles sobrecarregam ou excedem os recursos adaptativos da pessoa (Ballone, Ortolani e Neto, 2007). A construção de empreendimentos de petróleo e gás em territórios vulneráveis socialmente tem sido um exemplo comum de evento estressor na vida das populações do seu entorno devido à desfiguração do espaço e à mudança no cotidiano de vida dos territórios, geralmente provocada por uma urbanização acelerada e ausência de intervenção política em termos de melhorias em infraestrutura local (Seixas e Renk, 2011).

Um dos grandes empreendimentos petroquímicos do Brasil é o Complexo Petroquímico do Estado do Rio de Janeiro (Comperj), que estava sendo construído desde 2007 no município de Itaboraí, localizado na porção leste da região metropolitana do estado do Rio de Janeiro, mas teve suas obras 
cessadas em julho de 2015 (Souza, 2015). Esse contexto foi utilizado como estudo de caso, tendo em vista a importância do estímulo à percepção e à educação socioambiental com base na problematização da realidade.

As ações de educação ambiental devem ser precedidas por estudos de percepção socioambiental (Marin, 2008), que ainda são escassos quando aplicados ao contexto universitário da enfermagem (Camponogara et al., 2011; Bruzos et al., 2011). Contudo, é preciso ressaltar que a percepção ambiental de estudantes é permeada por valores, interesses, experiências e conhecimentos; não resulta apenas de informações instrumentalizadas em disciplinas curriculares ou outros momentos da vida acadêmica.

A percepção humana é aspecto cognitivo complexo e multidimensional, e sua relação com os fenômenos ambientais e de saúde demanda elementos de diversas áreas do conhecimento (ciências sociais, humanas, ambientais, da saúde etc.), pois se traduz em atitudes e transformações sociais (Marin, 2008). É preciso entender como futuros profissionais percebem a discussão da problemática ambiental contemporânea que afeta a saúde pública e respondem a ela, para que possam ser reformuladas as ações educativas e as matrizes curriculares dos cursos de graduação na direção da construção de um perfil acadêmico e profissional crítico, competente e participativo dessas questões.

De modo a subsidiar práticas pedagógicas socioambientais em espaços formativos do enfermeiro, o estudo aqui apresentado objetivou descrever a percepção de graduandos de enfermagem sobre impactos psicossociais e estratégias de coping de uma população vulnerabilizada pelo contexto de construção do Complexo Petroquímico do Rio de Janeiro.

\section{Metodologia}

Desenvolveu-se uma pesquisa-ação, descritiva, de abordagem qualitativa. As técnicas empregadas foram fotovoz e grupo focal, que têm sido utilizadas extensamente para capturar percepções e informações de indivíduos e grupos, com base na leitura do que os próprios atores interpretam como um problema por meio de suas fotografias e falas, respectivamente. A fotovoz foi selecionada para o estudo com a finalidade de se usarem as fotografias como pontos focais para as discussões do grupo e como pontos de apoio para a construção dos painéis, assim como na pesquisa de Tanjasiri e colaboradores (2011).

O desenvolvimento de todas as etapas da pesquisa-ação se deu no mês de maio de 2015. Havia 25 estudantes matriculados na disciplina enfermagem em saúde coletiva IV do quarto período do curso de graduação em enfermagem do Instituto de Humanidades e Saúde da Universidade Federal 
Fluminense (UFF), campus Rio das Ostras, no primeiro semestre de 2015. Desse universo, após ampla explicação do projeto e leitura do termo de consentimento livre e esclarecido, 18 estudantes concordaram em participar do estudo e assinaram o termo. O curso apresentava ainda uma matriz curricular em transformação e contava com quatro disciplinas relacionadas à área de enfermagem em saúde coletiva, mas nenhuma direcionada ao campo da saúde ambiental.

Os participantes realizaram visita ao município de Itaboraí até as áreas do entorno do Comperj. Foram orientados a fotografar as situações-problema do ambiente que interpretassem como impactos ambientais e psicossociais do contexto de construção do empreendimento na saúde das comunidades visitadas. O cenário real de conflitos e impactos ambientais do Comperj que foram gerados no município de Itaboraí em razão de sua fase de construção (Dias et al., 2013; Porto et al., 2014) se apresentou como um caso atual e próximo da realidade dos estudantes, por isso foi selecionado para o estudo.

As fotos foram armazenadas como arquivos digitais de imagem em CDs, selecionadas e impressas em datas anteriores à realização dos grupos focais. Em data posterior, com intervalo de duas semanas, realizou-se o grupo focal. Os participantes foram dispostos em círculo e identificados do número 1 ao 18. Em seguida, os participantes foram convidados a construir painéis integrados com base em colagem das seis fotos impressas em papel pardo grande e escrever, de forma sucinta, os impactos psicossociais observados. Cada participante emitiu sua justificativa sobre os impactos percebidos. As falas foram gravadas e transcritas e serviram, em conjunto com o painel, como múltiplos dados, processados com base nos objetivos do estudo e por meio da análise de conteúdo.

As categorias de análise identificadas foram: percepção de impactos psicossociais e estratégia defensiva coletiva. Em momento posterior, no espaço de ensino da disciplina enfermagem em saúde coletiva IV, realizou-se ação educativa para complementar os conhecimentos discentes, com base nos resultados do estudo.

A pesquisa foi parte integrante do projeto de pesquisa e extensão intitulado Impactos ambientais e à saúde do Comperj no município de Itaboraí/RJ: uso de tecnologia educacional para empoderamento de atores da educação e graduandos de enfermagem, que foi aprovado pelo Comitê de Ética em Pesquisa do Hospital Universitário Antônio Pedro, da Universidade Federal Fluminense, em dezembro de 2014, com o n. CAAE 39146814.5.0000.5243, e contemplado com recursos do Programa de Extensão Universitária do Ministério da Educação para 2015. 


\section{Resultados e discussões}

\section{Percepção dos impactos psicossociais}

Os impactos psicossociais relatados pelos participantes foram o desemprego; a elevação do número de casos de violência física e sexual; o aumento da criminalidade; e a elevação do consumo e tráfico de drogas. Os participantes ponderaram que tais impactos eram decorrentes de outros possíveis fatores ambientais: desordem urbana, favelização, deslocamento de mão de obra, aumento do contato de pessoas de fora da região com moradores locais, ausência de aplicação dos recursos em infraestrutura local.

A população criou muita expectativa achando que a cidade iria melhorar, fizeram muitos investimentos e não tiveram nenhum retorno, gerando muita ansiedade e podendo levar até mesmo à depressão (Participante 12, 24 anos).

(...) com o crescimento populacional que teve ali a estrutura urbana vai sendo prejudicada, porque as construções não são terminadas e os funcionários desse empreendimento, que muitas vezes vieram de outras cidades, permanecem em Itaboraí sem receber e sem ter como voltar pra casa. Com isso aumenta o índice de favelização, criminalidade, violência sexual, consumo e tráfico de drogas (Participante 4, 20 anos).

(...) se alguma construção vai ocorrer em determinado lugar, tem que ter um planejamento bem detalhado, porque depois de instalado isso vai ser refletido na população, e abandonar essa construção pela metade é abandonar a população, porque eles acabam dependendo disso. O que eu pude perceber é um descaso com aquele local (Participante 8, 26 anos).

Ansiedade e depressão foram relatadas pelos participantes como os possíveis problemas de saúde decorrentes da vivência dessas situações estressantes. Não houve relato sobre outros riscos à saúde (doenças cardiovasculares, imunológicas, câncer, transtornos mentais leves a severos etc.) produzidos pelo contato com os estressores socioambientais informados. Fatores psicológicos e socioambientais podem gerar reações adaptativas excessivas, sobrevindo processos patológicos físicos (Ballone, Ortolani e Neto, 2007).

Notou-se que havia clareza na percepção sobre a relação direta entre a oferta e qualidade dos atendimentos nos serviços de saúde e o aumento da demanda populacional provocado pela atração de mão de obra barata para a região. Os participantes informaram que não houve aumento do número 
de serviços e de profissionais da saúde na região, como deveria ter ocorrido na fase de implantação do empreendimento, o que provocou prejuízo nos atendimentos:

Quanto menor a renda daquelas pessoas, menos acesso à saúde elas terão, e isso agrava o surgimento de doenças, aumento da quantidade de filhos e diminuição do planejamento familiar (Participante 8, 26 anos).

Esse crescimento com certeza irá gerar um colapso no serviço de saúde, a não ser que aquilo que está no projeto do Comperj, que é levar infraestrutura pra toda a extensão onde o empreendimento está sendo construído, que isso realmente acontecesse e esse crescimento fosse planejado (Participante 2, 21 anos).

Falaram do Comperj como se fosse a maravilha do Brasil, com relação à geração de emprego, mas com relação à saúde a região em si não comportou todas as pessoas que foram à procura de emprego (Participante 7, 23 anos).

Esses dados são corroborados pelo estudo de Moniz e colaboradores (2014), que revela que a fala de atores da saúde em territórios do entorno do Comperj é consensual e indica que a região apresenta sérios problemas socioambientais e estruturais crônicos. No entanto, tal realidade se apresenta estacionária em relação à oferta e ao melhoramento de serviços públicos de saúde, educação, saneamento, transporte e segurança pública, durante a fase de construção do empreendimento.

Capacidade, disponibilidade e abrangência dos equipamentos urbanos básicos (saneamento ambiental, lazer, transporte público, serviços de saúde, escolas etc.) são quesitos fundamentais para garantir ambientes saudáveis e qualidade de vida. Assim, além da quantidade de estabelecimentos de saúde e educação, as competências e a abrangência estão relacionadas à qualidade de atendimento e à minimização do estresse da população; caracterizam-se como assuntos de suma importância que precisam ser debatidos durante as disciplinas dos cursos de graduação da área da saúde.

Souza (2011) verificou que as condições de mobilidade e a acessibilidade da população do entorno do Comperj aos equipamentos públicos de saúde de elevada complexidade (dois hospitais públicos) eram bastante precárias, em razão da inexistência de critérios de humanização e acolhimento, mobiliário urbano, posto policial próximo, sinalização e atendimento às normas de acesso para deficientes; baixa oferta de transporte público para os hospitais; deterioração de trechos, ausência de pavimentação e desníveis de pisos em calçadas e ruas; insuficiência na iluminação pública.

Contudo, outros impactos psicossociais produzidos pela situação atual de queda de incentivos fiscais, dos setores do comércio e imobiliário no 
município, interrupção das obras e grande número de demissões dos trabalhadores (Souza, 2015) não foram citados pelos estudantes como possíveis determinantes sociais a produzir sensação de mal-estar, conflitos e problemas psicológicos de ansiedade e preocupação excessiva com a deterioração das condições de ambiente, saúde e vida da população. Desse modo, a complexidade de impactos psicossociais não foi explorada em profundidade pelos participantes em razão da ausência de conhecimento prévio de elementos para discussão e fundamentação de suas percepções sobre a temática abordada.

As relações saúde-ambiente e saúde-trabalho precisam ser problematizadas e discutidas de forma transversal nos currículos e espaços formativos da enfermagem. Urge a formação de profissionais críticos e reflexivos que participem de ações que possam contribuir para a promoção da saúde e a melhoria das condições ambientais e de vida de indivíduos e grupos populacionais vulneráveis ambientalmente (Sena e Vaz, 2010; Bruzos et al., 2011).

\section{Estratégia defensiva coletiva}

Estratégias de enfrentamento aos estressores ambientais focadas na ação coletiva foram problematizadas por apenas dois participantes da pesquisa. Um deles citou a participação social em espaços decisórios de saúde e ambiente como importante estratégia defensiva coletiva nesse contexto.

A comunidade poderia participar e exigir os seus direitos fazendo reuniões, como as conferências públicas (Participante 17, 20 anos).

Outro participante destacou que não ter acesso à educação é um dos fatores responsáveis pela inexistência de monitoramento e controle social das obras do Comperj e seus impactos:

A falta de acesso à educação e às informações faz com que a população não fiscalize as obras do Comperj como deveria ser feito (Participante 18, 23 anos).

Embora não seja o único fator, o acesso à educação é um dos determinantes da atitude e do processo de transformação social. Não há como indivíduos ou grupos intervirem em seus meios sociais se não forem empoderados para isso por meio da educação.

No campo da saúde ambiental, a educação tem relevância fundamental ao estimular a consciência individual e coletiva sobre os fatores e as condições ambientais que contribuem para determinar ou condicionar a saúde humana; propulsionar o desenvolvimento de habilidades específicas e atitudes em 
prol de uma participação cidadã em relação à qualidade socioambiental em seu território (Loureiro, 2012; Jimenez e Terceiro, 2009).

A ausência de percepção da maioria dos estudantes sobre a participação social como um dos principais mecanismos coletivos de enfrentamento dos estressores ambientais reforçou um fato crítico: a necessidade da inclusão de uma educação ambiental que resulte em uma formação no mínimo político-social em espaços formativos do enfermeiro, como já ponderado em outros estudos (Camponogara et al., 2012, 2013).

Condições sociais como padrão de pobreza e acesso à educação podem ser relacionadas às dimensões de capacidade comunitária de acesso a recursos sociais e reflexão crítica sobre a situação ambiental e riscos à saúde. Assim, é mister que as principais dimensões da capacidade de organização e participação comunitária em espaços decisórios e de discussão sobre seus problemas socioambientais também sejam exploradas e aprofundadas na formação acadêmica da área da saúde. Tais dimensões são: liderança/representatividade; recursos e redes sociais e organizacionais; entendimento de sua história; poder; valores; reflexão crítica (Freudenberg, 2004).

Depreendeu-se que, para os respondentes, a culpabilização das situações-problemas atuais era meramente do governo local:

O governo deveria dar a essas pessoas melhores condições de vida. Na cidade de Itaboraí, eu percebi que várias ruas são de barro, isso sem contar as péssimas condições de moradia (Participante 9, 22 anos).

O governo não tem interesse mesmo em melhorar a infraestrutura da cidade: $o$ caminho pro Comperj é todo bonitinho, asfaltado, mas a cidade não tem infraestrutura, não tem saneamento básico, falta muita água, há anos não tem investimento em água lá (Participante 3, 23 anos).

Observou-se que os participantes não incluíram a própria população e os profissionais de saúde como corresponsáveis também pelas condições socioambientais discutidas. Esse dado foi corroborado pelo estudo de Camponogara e colaboradores (2013), que nos trouxe também a ausência de compreensão dos estudantes da área da saúde sobre o importante compromisso ético do profissional de participar socialmente com a/pela comunidade assistida em busca de ambientes saudáveis.

Diante do exposto, ressalta-se a importância de inserir e discutir em espaços acadêmicos temas relacionados à participação social, à atitude humana e às transformações socioambientais, uma vez que os impactos psicossociais prementes relacionados à segurança pública, aos riscos de violência e tráfico de drogas, dentre outros, exigem a necessidade de participação de toda a sociedade em espaços decisórios de saúde e ambiente. 
Para tanto, indivíduos trabalhadores e moradores precisam responder aos eventos estressores decorrentes das mudanças socioambientais cotidianas da vida de modo a desejarem buscar suporte social e resolução dos problemas. Notou-se que poucos foram os participantes que estabeleceram algum tipo de relação entre capacidade comunitária, atitude de resiliência e vulnerabilidade social de populações residentes no entorno de obras de grandes empreendimentos.

Indústrias de petróleo tendem a se localizar próximas a regiões onde residem populações vulneráveis socialmente, ou seja, que apresentam baixo nível de escolaridade e de participação social, portanto baixo poder contratual e de autonomia, elevado índice de pobreza e problemas de acesso a serviços básicos essenciais para a qualidade de vida (Moraes, 2010). O sofrimento que os impactos negativos da construção de grandes empreendimentos acarretam e as estratégias defensivas adotadas pelos grupos populacionais expostos a riscos socioambientais precisam alcançar maior visibilidade e discussão científica, política e social. Assim, conteúdos relativos ao fortalecimento da capacidade comunitária e às respostas de enfrentamento do estresse social e ambiental também precisam ser incluídos e tensionados de forma interdisciplinar em currículos integrados na área de formação da enfermagem, de modo a ajudar os futuros profissionais a desenvolver plenamente suas potencialidades para colaborar com as comunidades no sentido de enfrentar os impactos e obstáculos ao desenvolvimento sustentável e à promoção da saúde humana (Bruzos et al., 2011).

De forma geral, é preciso discutir com os estudantes a ideia de que os mecanismos de enfrentamento coletivo (confronto, suporte social, aceitação de responsabilidade, resolução de problemas e fuga, negação e esquiva etc.) (Dejours, 2015) podem decorrer da influência de fatores psicossociais, exposições ambientais, valores, experiências e informações dos impactos e dos benefícios dos projetos industriais (Luginaah et al., 2002).

\section{Considerações finais}

Concluiu-se que a estreita relação da dimensão social do ambiente no contexto específico de construção de um grande empreendimento petroquímico e a saúde mental não foi adequadamente estabelecida pelos participantes do estudo, uma vez que eles demonstraram desconhecimento de importantes determinantes socioambientais do processo saúde-doença e de sua relação com mecanismos de enfrentamento individual e coletivo. Faz-se necessária a inclusão de modo transversal da temática ambiental e sua dimensão psicossocial, que perpassam a vulnerabilização, o estresse e a capacidade de coping de populações aos impactos ambientais dos contextos de implantação 
e construção de processos industriais, nas disciplinas da graduação, pós-graduação e em outros espaços acadêmicos e de educação permanente do enfermeiro.

Sugere-se que novos estudos de percepção e educação ambiental e sua inter-relação com a saúde mental possam ser implementados no âmbito universitário da saúde e da enfermagem, com o propósito de aprimorar estratégias curriculares e pedagógicas que possibilitem a ampliação da capacidade política e do conhecimento ecológico do graduando de enfermagem. Tal movimento poderá fomentar o desenvolvimento de futuros profissionais enfermeiros para a compreensão dos fenômenos e a busca da melhoria das condições ambientais e de saúde mental de indivíduos e grupos assistidos em contextos de vulnerabilidade socioambiental.

\section{Agradecimento}

Ao apoio financeiro do Programa de Extensão Universitária (PROEXT) do Ministério da Educação.

\section{Colaboradoras}

As autoras participaram igualmente de todas as etapas de produção do manuscrito. 
Resumen El estudio buscó describir la percepción de estudiantes de pregrado de enfermería sobre impactos psicosociales y estrategias de coping de una población vulnerable por el contexto de construcción del Complejo Petroquímico de Río de Janeiro, Brasil. Se trató de una investigación acción, descriptiva, de enfoque cualitativo, realizada con 18 estudiantes de pregrado de enfermería por medio de la aplicación de las técnicas fotovoz y grupo focal durante el mes de mayo de 2015. Los datos recibieron tratamiento analítico de contenido. Los participantes destacaron apenas el consumo y tráfico de drogas, la violencia sexual, la disponibilidad de los servicios de salud como impactos sociales con gran potencial de producir problemas psicológicos, tales como ansiedad y depresión en la población, como consecuencias del proceso de construcción de la industria. Estrategias de coping a los estresores ambientales centradas en la acción colectiva fueron problematizadas por solo dos participantes. Se concluyó que la inclusión del modo transversal de la temática socioambiental - y su relación con la salud mental de poblaciones vulnerables a causa de los contextos de construcción de proyectos industriales en el país, en disciplinas de pregrado y otros espacios académicos - es fundamental, a fin de estimular la capacidad crítica, reflexiva y política de futuros enfermeros sobre la relación entre proyectos de desarrollo, degradación ambiental, equidad e impactos psicológicos en la población.

Palabras clave salud mental; salud ambiental; percepción; estudiantes de enfermería.

\section{Notas}

1 Universidade Federal Fluminense, Departamento de Enfermagem do Instituto de Humanidades e Saúde, Rio das Ostras, Rio de Janeiro, Brasil.

$<$ marceladeabreumoniz@gmail.com>

Correspondência: Rua Recife, s/n, CEP 28895-532, Jardim Bela Vista, Rio das Ostras, Rio de Janeiro, Brasil.

2 Universidade Federal Fluminense, Especialização/Residência em Enfermagem em Saúde Coletiva, Niterói, Rio de Janeiro, Brasil.

<jaque.manhaes@hotmail.com>

3 Universidade Federal Fluminense, Curso de Graduação em Enfermagem, Rio das Ostras, Rio de Janeiro, Brasil.

<rayaramozer@gmail.com> 


\section{Referências}

BALLONE, Geraldo J.; ORTOLANI, Ida V.; NETO, Eurico P. Da emoção à lesão: um guia de medicina psicossomática. 2. ed. São Paulo: Manole, 2007.

BRUZOS, Gabriela A. S. et al. Meio ambiente e enfermagem: suas interfaces e inserção no ensino de graduação. Saúde e Sociedade, São Paulo, v. 20, n. 2, p. 462-469, abr.-jun. 2011.

CAMPONOGARA, Silviamar et al. Enfermagem e meio ambiente: uma revisão bibliográfica. Revista de Enfermagem da UFSM, Santa Maria, v. 1, n. 3, p. 472-480, set.-dez. 2011.

CAMPONOGARA, Silviamar et al. The perception of students of the area of health about the environmental problems: a descriptive study. Online Brazilian Journal of Nursing, Niterói, v. 11, n. 2, ago. 2012. Disponível em: <www.objnursing.uff.br/ index.php/nursing/article/view/3767>. Acesso em: 30 jul. 2015.

CAMPONOGARA, Silviamar et al. Saúde e meio ambiente: subsídios para reflexão sobre a formação acadêmica na área da saúde. Ciência, Cuidado e Saúde, Maringá, v. 12, n. 3, p. 564-571, jul.-set. 2013.

DEJOURS, Christophe. A loucura do trabalho: estudo de psicopatologia do trabalho. 6. ed. São Paulo: Cortez, 2015.

DIAS, Alexandre P. et al. Complexo Petroquímico do Rio de Janeiro (Comperj): impactos socioambientais, violação de direitos e conflitos na Baía de Guanabara. Revista Ética e Filosofia Política [on line], Juiz de Fora, v. 1, n. 16, p. 151-175, 2013. Disponível em: <www.ufjf.br/eticaefilosofia/ files/2009/08/16_1_pessoa.pdf > . Acesso em: 11 jan. 2015 .

FREUDENBERG, Nicholas. Community capacity for environmental health promotion: determinants and implications for practice. Health Education \& Behavior, New York, v. 31, n. 4, p. 472-490, ago. 2004.
HASSAN, Amir A. et al. Environmental health impact assessment of development projects: a practical guide for the WHO Eastern Mediterranean Region. Amman: Liverpool School of Tropical Medicine, Islamic Development Bank, World Health Organization Regional Office for the Eastern Mediterranean Regional Centre for Environmental Health Activities, Arab Gulf Programme for United Nations Development Organizations, 2005.

JIMENEZ, Susana; TERCEIRO, Emanoela. A crise ambiental e o papel da educação: um estudo fundado na ontologia marxiana. Educação em Revista, Belo Horizonte, v. 25, n. 3, p. 299-325, dez. 2009.

LOUREIRO, Carlos F. B. Sustentabilidade e educação: um olhar da ecologia política. São Paulo: Cortez, 2012.

LUGINAAH, Isaac N. et al. Community responses and coping strategies in the vicinity of a petroleum refinery in Oakville, Ontario. Health Place, Edinburgh, v. 8, n. 3, p. 177-190, set. 2002.

MARIN, Andreia A. Pesquisa em educação ambiental e percepção ambiental. Pesquisa em Educação Ambiental, Ribeirão Preto, v. 3, n. 1, p. 203-222, 2008.

MONIZ, Marcela A. et al. Percepção de impactos socioambientais no contexto da implantação do Comperj no município de Itaboraí. In: CONGRESSO NACIONAL DE MEIO AMBIENTE DE POÇOS DE CALDAS, 11., 2014. Anais (on line)... Poços de Caldas: GSC Eventos Especiais, Instituto Federal de Educação, Ciência e Tecnologia do Sul de Minas Gerais, 2014. Disponível em: <http://meioambientepocos.com.br/portal/anais/2014/index.php>. Acesso em: 20 nov. 2015.

MORAES, Ana C. L. Condições de saúde e ambiente no entorno de um polo petroquímico no Rio Grande do Norte: uma análise integrada. 179 f. Tese (Doutorado em Ciências na área de Saúde Pública e Meio Ambiente) - Escola 
Nacional de Saúde Pública Sergio Arouca, Fundação Oswaldo Cruz, Rio de Janeiro, 2010.

PORTO, Marcelo F. et al. Mapa de conflitos envolvendo injustiça ambiental e saúde no Brasil, 2014. Disponível em: <www. conflitoambiental.icict.fiocruz.br $>$. Acesso em: 13 fev. 2015.

SEIXAS, Sônia R. C.; RENK, Michelle. Projetos do setor de petróleo e gás no Sudeste brasileiro: algumas considerações sobre o desafio desenvolvimento x preservação ambiental. In: SELENE, H. Oficina sobre Impactos sociais, ambientais e urbanos das atividades petroliferas: o caso de Macaé. Niterói: Programa de Pós-Graduação em Sociologia e Direito da Universidade Federal Fluminense, 2011. p. 443-456.

SENA, Janaína; VAZ, Marta R. C. A relação saúde/ambiente nos processos de formação do profissional enfermeiro: um ensaio teórico. Revista Eletrônica do Mestrado em Educação Ambiental, Rio Grande, v. 24, p. 265-273, jan.-jun. 2010. Disponível em: <www.seer. furg.br/remea/article/view/3900>. Acesso em: 07 jan. 2017.
SOUZA, Bárbara. Com projeto original do Comperj descartado, Itaboraí enfrenta crise nos empreendimentos. Jornal da $C B N$, Rio de Janeiro, 8 jul. 2015. Disponível em: $<$ http://cbn.globoradio.globo.com/series/ itaborai-desilusao-pos-comperj/2015/07/08/ COM-PROJETO-ORIGINAL-DO-COMPERJDESCARTADO-ITABORAI-ENFRENTACRISE-NOS-EMPREENDIMENTOS.htm \#ixzz3hsbVhnpP>. Acesso em: 8 jul. 2015.

SOUZA, Rossana S. Infraestrutura e saúde: elementos para uma abordagem multidisciplinar. 126 f. Dissertação (Mestrado em Arquitetura e Urbanismo) - Faculdade de Arquitetura, Universidade Federal Fluminense, Niterói, 2011.

TANJASIRI, Sora P. et al. Using photovoice to assess and promote environmental approaches to tobacco control in AAPI communities. Health Promotion Practice, Chicago, v. 12, n. 5, p. 654-665, set. 2011.

Recebido em 30/10/2015

Aprovado em 14/04/2016 